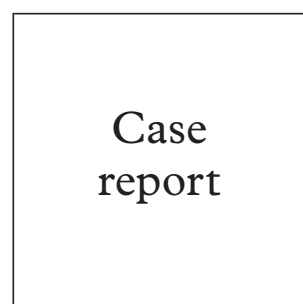

Department of Sexual Medicine, Birmingham

Heartlands Hospital, Birmingham B9 5SS UK

D J White

A R Habib

A Vanthuyne

Pharmacy

Manufacturing Unit, North Staffordshire Hospital, Stoke on

Trent ST4 6QG, UK $S$ Langford

\section{Department of Pharmacy, Birmingham \\ Heartlands Hospital, Birmingham \\ M Symonds}

Correspondence to: D J White, Department of Sexual Medicine, Hawthorn House, Heartlands Hospital, Birmingham B9 5SS, UK dwhite@hawthorn.co.uk or A R Habib

ahabib@hawthorn.co.uk

Accepted for publication 22 March 2001

\title{
Combined topical flucytosine and amphotericin B for refractory vaginal Candida glabrata infections
}

\author{
D J White, A R Habib, A Vanthuyne, S Langford, M Symonds
}

Patients with vaginitis due to highly azole resistant Candida glabrata can be particularly difficult to treat. We describe three cases of longstanding vaginal candidiasis due to $C$ glabrata. These had failed to respond to local and systemic antifungals. Flucytosine (1 g) and amphotericin B (100 $\mathrm{mg}$ ) formulated in lubricating jelly base in a total $8 \mathrm{~g}$ delivered dose, was used per vagina once daily for 14 days with significant improvement, both clinically and microbiologically. (Sex Transm Inf 2001;77:212-213)

Keywords: amphotericin; flucytosine; Candida glabrata

\section{Introduction}

Candida glabrata is the second most common yeast recovered from the genital tract of women with vaginitis and accounts for about $5 \%$ of vaginal infections. ${ }^{1}$ A substantial minority of $C$ glabrata isolates are azole resistant $^{2}{ }^{3}$ and further resistance may be selected out by noncurative treatment. Although infections with this organism are not always associated with symptoms and clinical signs ${ }^{4}$ some affected women have discharge and/or vulvitis and a poor response to antifungal therapy. ${ }^{56}$

We describe three cases of persistent vaginal candidiasis due to $C$ glabrata, unresponsive to conventional antifungal therapy including boric acid. Flucytosine tablets $500 \mathrm{mg}$ (Center Specialites Pharmaceutiques Cournon Cedex, France) and amphotericin B BP1998 $1 \mathrm{mg}=$ 859IU (Bufa BV Uitgeest Holland) were combined in lubricating jelly, Aquagel (Adams Healthcare, UK). This was used per vagina with clinical and microbiological cure. Treatment was delivered by a unit dose vaginal applicator containing amphotericin $100 \mathrm{mg}+$ flucytosine $1 \mathrm{~g}$ based in Aquagel in a total $8 \mathrm{~g}$ delivered dose. This preparation has an unknown shelf life and is obtainable from the pharmacy manufacturing unit, North Staffordshire Hospital, Stoke on Trent ST4 6QG (tel 01782552 289; fax 01782552 916). The preparation was compounded no more than 48 hours before treatment began.

\section{Case 1}

A 42 year old woman presented with "recurrent vaginal thrush" since the age of 19 . At referral she had had several recent swabs showing heavy growths of candida species in Gram stain microscopy despite 3 months' treatment with itraconazole $100 \mathrm{mg}$ twice daily for 2 days every week, two Depo-provera (Pharmacia \& Upjohn) injections, and a number of other unspecified antifungal treatments including courses of nystatin pessaries. None of these produced any symptomatic response.

Vaginal swabs were positive by Gram stain and culture for $C$ glabrata which persisted despite itraconazole $200 \mathrm{mg}$ once daily for 14 days combined with clotrimazole $500 \mathrm{mg}$ vaginal pessaries for 7 nights, intravaginal painting with gentian violet $0.5 \%$ aqueous solution for 3 days, and boric acid $600 \mathrm{mg}$ in gelatine capsules once daily for 14 nights.

Intravaginal amphotericin B and flucytosine in lubricating jelly was given at night for 14 days. Her symptoms improved and Gram stain and cultures were negative 2 and 5 weeks following treatment.

\section{Case 2}

A 63 year old woman presented with a 6 year history of intermittent vulvo-vaginitis and persistent isolation of $C$ glabrata on Gram stain and culture. This had failed to respond to a variety of differing types and lengths of azole therapy. She had had a hysterectomy 5 years before presentation following which she commenced subcutaneous oestrogen hormone replacement implants. One year after the hysterectomy her symptoms of vaginal discharge and vulval soreness became continuous. She had had some minor symptomatic improvement to dydrogesterone and intravaginal boric acid.

She was treated with itraconazole $100 \mathrm{mg}$ daily combined with nystatin pessaries for 4 weeks followed by intravaginal boric acid for 2 weeks. Despite this, Gram stain and cultures remained positive for $C$ glabrata. This was fully sensitive to antifungals in vitro (table 1 ).

Amphotericin B and flucytosine in lubricating jelly was given once daily for 14 days. She improved symptomatically and follow up swabs were negative by Gram stain microscopy and culture 3, 4, and 8 weeks afterwards. Seven

Table 1 Antifungal sensitivities (NCCLS method Bristol PHLS mycology reference laboratory) before treatment with intravaginal flucytosinelamphotericin B in lubricating gel

\begin{tabular}{llll}
\hline & Case 1 & Case 2 & Case 3 \\
\hline Amphotericin B & $\mathrm{S}$ & $\mathrm{S}$ & - \\
Flucytosine & $\mathrm{S}$ & $\mathrm{S}$ & - \\
Fluconazole & $\mathrm{R}$ & $\mathrm{S}$ & $\mathrm{R}$ \\
Itraconazole & $\mathrm{R}$ & $\mathrm{S}$ & $\mathrm{R}$ \\
Miconazole & - & $\mathrm{S}$ & - \\
Nystatin & - & $\mathrm{S}$ & -
\end{tabular}

$\mathrm{S}=$ sensitive, $\mathrm{R}=$ resistant. 
months later she presented with a 4 week history of discharge. Microscopy of a Gram stained slide was positive for spores and a nonalbicans yeast (not further speciated) was isolated in culture. She responded symptomatically and microbiologically to nystatin pessaries at night for 14 nights.

\section{Case 3}

A 42 year old woman presented with intractable symptoms of "vaginal thrush" which had started since a hysterectomy 1 year earlier, following which she had started unopposed oestrogen hormone replacement therapy.

Microscopy of a Gram stained vaginal slide showed spores and $C$ glabrata was isolated which persisted despite dydrogesterone $10 \mathrm{mg}$ daily for 28 days combined with nystatin pessaries at night for 14 days, combined nystatin pessaries and itraconazole $400 \mathrm{mg}$ daily for 7 days, and vaginal boric acid $600 \mathrm{mg}$ daily for 14 days.

Intravaginal amphotericin B and flucytosine in lubricating jelly was given at night for 14 days. Her symptoms improved and microscopy and cultures were negative 2 and 5 weeks following treatment.

\section{Discussion}

By comparison with $C$ albicans, $C$ glabrata is intrinsically less sensitive to azole antifungals and, because this organism is haploid (unlike $C$ albicans which is diploid) selection of drug resistant strains may occur. ${ }^{7}$ Persistent vaginal $C$ glabrata is more likely to be found in patients who are clinically not or partially responsive to azole antifungals, older patients, diabetics, and women who have had hysterectomies. ${ }^{4}$ Symptoms are, however, not a reliable guide to the causative organism. It is therefore important to speciate isolates from patients presenting with problem vaginal candidosis. ${ }^{4}$ Because of the relatively small numbers of patients presenting with this condition, treatment of persistent $C$ glabrata vaginitis is not evidence based but remains largely anecdotal. Most clinicians would start treatment with intravaginal nystatin (the only licensed alternative to azoles in the United Kingdom) and then proceed to either high dose oral itraconazole together with high dose intravaginal azole pessaries or nystatin. Following this with intravaginal boric acid 600 $\mathrm{mg}$ at night for 14 days. ${ }^{8}$ If this fails however there has previously been no further treatment available. Intravaginal amphotericin/ flucytosine offers a possible treatment for such patients.

Topical flucytosine has been used for vaginal infections caused by both $C$ albicans ${ }^{9}$ and antifungal resistant non-albicans candidiasis ${ }^{10}{ }^{11}$ but a suitable formulation has not been available in the United Kingdom. Although it is the only available fungicidal agent, ${ }^{1}$ there are reservations about its topical use because of the potential development of flucytosine resistance, which occurs by mutation of a single gene. The risk of such resistance developing is thought to be reduced by combination with polyene antifungals such as amphotericin B with which flucytosine is synergistic in vitro. ${ }^{19}$

Our three cases demonstrate that flucytosine and amphotericin in lubricating jelly may be effective in chronic vaginal $C$ glabrata infection where all other available agents have failed. This treatment was well tolerated in all patients with no or minimal side effects.

We would like to thank Professor Frank Odds for his initial advice and Dr Elizabeth Johnson for help with the results of the antifungal sensitivity tests.

Contributors: DJW, ARH, and AV collected the patients and wrote the paper; DJW had the idea of using flucytosine and amphotericin intravaginally; MS and SL developed the formulation of amphotericin and flucytosine in lubricating jelly.

1 Odds FC. Candida and candidosis. 2nd ed. London: Bailliere Tindall, 1988

2 Lynch ME, Sobel JD. Comparative in vitro activity of antimycotic agents against pathogenic vaginal yeast isolates. $\mathcal{F}$ Med Vet Mycol 1994;32:267-74.

3 Otero L, Fleites A, Mendez FJ, et al. Susceptibility of Candida species isolated from female prostitutes withlpar vulvovaginitis to antifungal agents and boric acid. Eur $\mathcal{F}$ Vlin Microbiol Infect Dis 1999;18:59-61.

4 Geiger AM, Foxman B, Sobel JD. Chronic vulvovaginal candidiasis: characteristics of women with Candida albicans, C glabrata and no candida. Genitourin Med 1995;71: cans, $304-7$

5 Redondo-Lopez V, Lynch M, Schmitt C, et al. Torulopsis glabrata vaginitis: clinical aspects and susceptibility to antiungal agents. Obstet Gynecol 1990;76:651-5.

6 White DJ, Johnson EM, Warnock DW. Management of persistent vulvo-vaginal candidosis due to azole-resistant Candida glabrata. Genitourin Med 1993;69:112-14.

7 Kerridge D, Nicholas RO. Drug resistance in the opportunistic pathogens Candida albicans and Candida glabrata. $\mathcal{F}$ Antimicrob Chemother 1986;18:39-49.

8 Bingham JS. What to do with the patient with recurrent vulvovaginal candidiasis. Sex Transm Inf 1999;75:225-7.

9 Larsson-Cohen U. Treatment of vaginal candidosis with a single dose of a new antimycotic drug, 5-fluorocytosine + Candicidin. Castellania 1977;5:81-2.

10 Horowitz BJ. Topical flucytosine therapy for chronic recurHorowitz BJ. Topical flucytosine therapy for chronic recur-
rent Candida tropicalis infections. $\mathcal{F}$ Reprod Med 1986;31: $821-4$.

11 Fidel PLJ, Vazquez JA, Sobel JD. Candida glabrata: review of epidemiology, pathogenesis, and clinical disease with comparison to C. albicans. [Review] [185 refs]. Clin Microbiol Rev 1999;12:80-96. 